\title{
Cytokines and Their STATs in Cutaneous and Visceral Leishmaniasis
}

\author{
Hannah E. Cummings, ${ }^{1}$ Rashmi Tuladhar, ${ }^{1}$ and Abhay R. Satoskar, ${ }^{1,2}$ \\ ${ }^{1}$ Department of Microbiology, The Ohio State University, 484 West 12th Avenue, Columbus, OH 43210, USA \\ ${ }^{2}$ Center for Microbial Interface Biology, The Ohio State University, 484 West 12th Avenue, Columbus, OH 43210, USA
}

Correspondence should be addressed to Abhay R. Satoskar, satoskar.2@osu.edu

Received 9 September 2009; Accepted 18 November 2009

Academic Editor: Luis I. Terrazas

Copyright () 2010 Hannah E. Cummings et al. This is an open access article distributed under the Creative Commons Attribution License, which permits unrestricted use, distribution, and reproduction in any medium, provided the original work is properly cited.

Cytokines play a critical role in shaping the host immune response to Leishmania infection and directing the development of protective and non-protective immunities during infection. Cytokines exert their biological activities through the activation and translocation of transcription factors into the nucleus whether they drive the expression of specific cytokine-responsive genes. Signal transducer and activator of transcription (STATs) are transcription factors which play a critical role in mediating signaling downstream of cytokine receptors and are important for shaping the host immune response during Leishmania infection. Here we discuss the signature cytokines and their associated STATs involved in the host immune response during cutaneous and visceral leishmaniasis.

\section{Introduction}

Leishmaniasis is a zoonotic disease resulting from infection with protozoan parasites of the species Leishmania, of which approximately 30 different species have been described [1] The clinical manifestations of disease differ depending on causative species and occur in three major forms: cutaneous, mucosal, and visceral leishmaniasis [1-3].

Leishmania infection is transmitted to susceptible mammalian hosts by sand flies of the genus Phlebotomous and Lutzomyia in the Old and New World, respectively [3]. Leishmania parasites exist as an extracellular promastigote within the gut of the sand fly, transforming into an intracellular amastigote within macrophages of mammalian hosts. As obligate intracellular pathogens in mammals, parasite survival and replication requires that parasites are rapidly internalized by host cells. While macrophages are considered to be critical effector cells in eliminating parasites and resolving disease, these cells play an important role during infection as the definitive host cell in which Leishmania species survive and replicate [4].

The genetic background of the host plays an important role in determining host resistance or susceptibility and dis- ease outcome [5]. T lymphocytes play critical role in shaping the host immune response by secreting cytokines, which may act both synergistically and antagonistically through complex signaling pathways to direct both protective and non-protective immunities against intracellular parasites. Early studies using mouse models of experimental CL have revealed a clear dichotomy between Th1-associated cytokines mediating protection and Th2-associated cytokines mediating susceptibility $[5,6]$. On the other hand during VL, Th2 response and cytokines such as IL-4 and IL-13 seem to be necessary for immunity and efficient response to antileishmanial chemotherapy $[7,8]$.

The ability of the host to control infection and resolve disease requires the generation of cell-mediated immune responses capable of activating host macrophages to eliminate intracellular parasites. In both human and experimental models of CL, control of infection is mediated by $\mathrm{T}$ lymphocytes and is critically dependent upon the early induction of an IL-12-driven Th1-type immune response and the production of IFN- $\gamma$ by CD4+ T cells $[5,9]$. IFN$\gamma$ plays a critical role in the activation of macrophages to kill intracellular parasites by inducing production of nitric oxide which is critical for elimination of parasites [6]. 
Previous studies have shown that susceptibility to infection and disease progression in CL is driven largely by the induction of a non-protective, IL-4-driven Th2-type immune response and the production of Th2-associated cytokines IL-4, IL-10, IL-13, and TGF- $\beta$ [9-12]. In experimental models of murine CL, IL-4 was shown to play a critical role in mediating susceptibility to Leishmania infection by down-regulating the expression of protective Th1-associated cytokines IL-12 and IFN- $\gamma$ and by inhibiting NO production and parasite killing by macrophages [6]. Nonetheless, some studies using IL-4-deficient BALB/c mice have shown that IL-4-mediated exacerbation of CL is dependent upon the particular strain of Leishmania [13]. IL-10 and TGF- $\beta$ have been shown both in human and experimental models of disease to suppress healing responses and prevent parasite clearance by the host $[12,14-16]$.

The clear Th1/Th2 dichotomy observed in murine cutaneous leishmaniasis has not been demonstrated in humans, as the factors governing the nature of the host immune response and disease outcome during leishmaniasis are far more complex in men than in mice [14]. It is clear however, in both human and experimental models of disease, that cytokines play a critical role in shaping the nature of the host immune response in response to Leishmania infection.

Cytokines signal through a cascade of intracytoplasmic proteins known as STATs (signal transducer and activator of transcription) [17]. These transcription factors are indispensable for mediating biological activities of cytokines and for induction of cytokine-responsive genes. STATs are activated by tyrosine phosphorylation by cytokine receptor-associated Jak kinases. Upon activation, the STATs are phosphorylated and dimerize through their $\mathrm{SH} 2$ domain to form homo- or heterodimers. These dimers translocate to the nucleus where they bind to the promoter region of genes via specific DNA binding domains and thus bring about the transcription of their respective genes [17-20].

Here, we discuss the signature cytokines and complex STAT-mediated signaling networks involved in regulating the host response and determining disease outcome during cutaneous and visceral leishmaniasis.

\section{Interferon- $\gamma($ IFN- $\gamma)$ and STAT1}

The development of cell-mediated immune responses capable of controlling Leishmania infection and resolving disease is critically dependent upon signaling by interferon gamma (IFN- $\gamma$ ), a type II interferon secreted primarily by activated $\mathrm{T}$ cells and NK cells in response to IL-12 signaling [21]. The major biological function of IFN- $\gamma$ is to activate macrophages and enhance the microbicidal activity of these cells to kill intracellular pathogens [22]. IFN- $\gamma$ induces iNOS (inducible nitric oxide synthase) expression and NO production by phagocytes harboring intracellular parasites and is required for activating macrophages to eliminate parasites and resolve Leishmania infection $[9,10]$.

The biological effects of IFN- $\gamma$ are largely dependent upon the activation of STAT1 transcription factors. Upon IFN- $\gamma /$ IFN $\gamma$ R ligation, STAT1 is phosphorylated and STAT1 homodimers associate and translocate into the nucleus to initiate gene transcription [21]. IFN- $\gamma$-dependent immune responses against microbial pathogens are severely impaired in mice genetically deficient in STAT1 [23]. In experimental models of cutaneous L. major infection, STAT1 ${ }^{-/}$mice developed extensive tissue destruction and severe inflammatory infiltrates composed heavily of neutrophils and parasitized macrophages [24]. STAT1/IFN- $\gamma$ signaling pathway also induces the expression of Th1-associated transcription factor, T-bet. Both STAT1 and T-bet are required to mount an efficient Th1 response and as such, are indispensable for host defense against Leishmania infection in mice [19, 25]. In contrast, STAT $1^{-/-}$mice are resistant to visceral infection with $L$. donovani even in the absence of a strong Th1 response and develop minimal liver pathology. Studies in these mice have opened the possibility that resistance in STAT $1^{-/-}$mice is due to a defect in the trafficking of monocytes and an inability of parasites to establish a successful infection [19]. In fact, a recent study by our group has shown that STAT1 in CD4+ T cells plays a critical role in immunity to L. major by mediating recruitment of T cells to the site of infection [26]. This is perhaps not surprising since STAT1 is required for efficient induction of $\mathrm{C}-\mathrm{X}-\mathrm{C}$ chemokine receptor 3 (CXCR3) on CD4+ T cells [27] which is critical for efficient recruitment to the site of infection [28]. Interestingly, STAT1 in T cells is not necessary for Th1 development and IFN- $\gamma$ production during L. major infection $[26,29]$.

\section{Interferon- $\alpha / \beta($ IFN $-\alpha / \beta)$ and STAT 2}

Type I interferons $\alpha$ and $\beta($ IFN- $\alpha / \beta)$ are proinflammatory cytokines that signal through the activation and phosphorylation of STAT1 and STAT2. Studies using mice deficient of STAT1 and STAT2 have shown that both these transcription factors are required for mediating most of the proinflammatory effects of these cytokines [30]. In cutaneous infection with $L$. major, INF- $\alpha / \beta$ have been shown to act as early regulators of the innate response to infection and are required for initiating the expression of nitric oxide synthase type 2 (NOS2) and the production of NO [30]. The expression of NOS2 by macrophages has been shown to play an important role in protection against intracellular parasites such as cryptococci, Toxoplasma, mycobacteria, and Leishmania. A study by Bogdan et al. found that type I IFN $-\alpha / \beta$ are required for initial NOS2 expression [30]. Via NOS2, IFN $\alpha / \beta$ play a critical role in the innate response to $\mathrm{CL}$ infection by mediating events involved in parasite containment, IFN- $\gamma$ expression, and the activity of cytotoxic NK cells [30].

Mice deficient of STAT2 are known to demonstrate an increased susceptibility to viral infections and impaired responsiveness to type I interferons [31]. In CL caused by $L$. amazonesis, infection with intracellular amastigotes was found to reduce STAT2 phosphorylation and enhance STAT2 degradation through the activity of parasite-derived proteases in DC from infected $\mathrm{C} 57 \mathrm{BL} / 6$ and $\mathrm{BALB} / \mathrm{c}$ mice. The role of STAT2 in VL is largely unknown [31]. 


\section{Interleukin-12 (IL-12) and STAT4}

The proinflammatory cytokine, IL-12, is a heterodimer composed of two subunits, p35 and p40 and is produced primarily by macrophages and dendritic cells (DCs) in response to microbial pathogens [32]. IL-12 functions as the main physiological inducer of gamma interferon (IFN$\gamma$ ) by activated $\mathrm{T}$ cells and promotes Th1-type CD4+ T cell differentiation, and therefore plays an important role in inducing cell-mediated protection in response to Leishmania infection [33].

The importance of IL-12 in immunity to CL has been clearly demonstrated in experimental models of L. major infection: animals genetically deficient in the IL-12 gene or genetically resistant mice treated with anti-IL-12 antibodies fail to control parasite replication and are unable to resolve infection, and treatment of genetically susceptible BALB/c mice with recombinant IL-12 is sufficient to confer resistance in these animals [34]. Similarly, a comparison between L. donovani-infected IL-12 $12^{+/+}$and IL-12 $2^{-/-}$C57BL/6 mice showed a higher parasitic burden in the livers of infected IL$12^{-/-}$mice [35].

The specific cellular effects of IL-12 are due to the activation of Janus kinase (JAK)-STAT pathways, primarily to the activation of the specific transcription factor, STAT4 [32]. STAT4 is one of seven members of the STAT family of transcription factors and is the major STAT activated by IL12 [33]. In activated T cells and NK cells, STAT4 functions to induce IFN- $\gamma$ production in response to IL-12 signaling. IL12 signaling leads to activation of STAT4 by Jak kinase Jak2 and Tyk2. STAT4 dimers translocate into the nucleus and bind DNA sequences at IFN- $\gamma$ activation site (GAS) $[17,18]$. IL-12-induced IFN- $\gamma$ mediates most of the proinflammatory activities of IL-12 and is critical for Th1 differentiation and directing the cell-mediated immune response required for protection against Leishmania infection.

STAT4-deficient mice are viable but have impaired Th1 differentiation, IFN- $\gamma$ production, and cell-mediated immunity, phenotypes shared by mice lacking IL-12 or IL$12 \mathrm{R}$ subunits [33]. In experimental models of cutaneous leishmaniasis, STAT4-dependent IL-12 signaling was found to be crucial for the development of protective immunity as evidenced by the increased susceptibility observed in STAT4deficient mice to L. major [32], and STAT4/STAT6 ${ }^{-/}$mice to L. mexicana [36].

\section{IL-4, IL-13 and STAT6}

IL-4 and IL-13 are the signature cytokines associated with Th2-type immune responses and are associated with nonhealing forms of cutaneous disease in mice [9]. IL-4 stimulates the differentiation of naïve $\mathrm{CD} 4+\mathrm{T}$ cells into Th2 cells capable of producing Th2-associated cytokines IL-5, IL-10 and IL-13, and promotes antibody production and IgE class switching by B cells. IL- 4 also functions as a powerful inhibitor of IFN- $\gamma$-producing CD4+ T cells and suppressor of protective Th1 immune responses [37]. IL-4, along with related cytokine IL-13, trigger macrophages to undergo alternative activation and is associated with parasite survival and persistence of infection $[9,10,24]$.

The IL-4R and IL-13R share a common $\gamma \mathrm{c}$ receptor chain involved in signal transduction. Both IL-4 and IL-13 are the principal activators of transcription factor STAT6 which mediates most biological activities of these cytokines [38]. STAT6 $^{-1-}$ mice show impaired Th2 differentiation and lose responsiveness to IL-4 and IL-13 while maintaining normal responses to other cytokine signals [23].

The importance of IL-4, IL-13, and STAT6 in mediating susceptibility during cutaneous infection was clearly demonstrated in experimental models of $L$. mexicana infection using genetically susceptible mice deficient in the genes for IL-4, IL-13, and STAT6. IL-4 ${ }^{-/-}$, IL-13 ${ }^{-/-}$, IL-4 $4^{-/-} / \mathrm{IL}-$ $13^{-/-}$, and STAT6 $6^{-/-}$mice all mount a robust Th1 response and effectively control parasite growth and replication, indicating that IL-4, IL-13, and STAT6 mediate susceptibility by preventing the development of protective Th1 responses $[5,39]$.

L. donovani-infected mice have been demonstrated to produce IL-4 in some models and not in others [40, 41]. However, IL-4 does not seem to play a part in promoting susceptibility to $L$. donovani as susceptible phenotypes have been shown even in the absence of IL-4 [41]. In experimental models of visceral infection in mice, IL-4 has been found to play no antagonistic role in eliminating infection [40]. Some studies have actually shown that IL- $4^{-/-}$mice are more susceptible to infection with $L$. donovani than wild type mice and suggest a protective role for IL-4 in VL $[8,39]$. Since IL-4 does not seem to exacerbate $L$. donovani infection, it appears that $\mathrm{Th} 2$ responses are not important in suppressing the protective Th1 immune response. Furthermore, the assumption that treatment of VL should be enhanced in absence of IL-4 was shown not to be true. In fact, $L$. donovani-infected IL- $4^{-/-}$mice were seen to be as susceptible to infection as wild type mice. Rather, resolution of infection upon drug treatment was more effective in wild type mice as compared to IL4 ${ }^{-/-}$mice [8]. The findings suggest a role for IL-4 in enhancing the protective role of IFN- $\gamma$. Experimental studies in $\mathrm{BALB} / \mathrm{c}$ mice genetically deficient in IL-4 were found to respond poorly to chemotherapy with sodium stibogluconate and these mice were also found to produce little IFN- $\gamma$. A recent study has shown that IL- 4 plays a critical role in vaccine induced protection against VL by enhancing IFN $-\gamma$ production by $\mathrm{CD} 8+\mathrm{T}$ cells [42].

\section{IL-10 and STAT2}

The anti-immune and anti-inflammatory cytokine, IL-10 is produced by a variety of cells including $\mathrm{T}$ cells, monocytes, macrophages, DCs, and B cells [15]. While many cells can produce IL-10, the main biological functions of IL-10 appear to be on macrophages and DCs. IL-10 functions to inhibit the production of proinflammatory cytokines IL-1, IL-6, IL-12, and tumor necrosis factor (TNF) by macrophages and DCs [23] and thus prevents the expansion of Th1-type cells required for protective immunity during Leishmania infection [14-16]. 
IL-10 also promotes activation, survival, and antibody production by $\mathrm{B}$ cells and the development of humoral immune responses which play a detrimental role in host defense against Leishmania infection by facilitating parasite entry into host cells [43]. In both human and experimental models of CL, high levels of IL-10 production are strongly associated with nonhealing forms of disease $[9,15]$.

In the absence of IL-10 (IL-10 $0^{-/}$), genetically susceptible C57BL/6 mice mount an immune response capable of controlling parasite replication and resolving cutaneous infection with $L$. mexicana. IL- $10^{-/-}$mice were found to express higher levels of IFN- $\gamma$ and produced more NO than C57BL/6 IL-10 ${ }^{+/+}$mice. IL-10 also mediates susceptibility and promotes parasite persistence in cutaneous infections with $L$. major, however its role in $L$. amazonensis and $L$. pifanoi infection remains unclear [15].

Increased IL-10 production has been reported in patients suffering from $\mathrm{VL}$ as well as mice infected with $L$. donovani [44]. IL-10 levels decline upon resolution of VL following chemotherapy indicating that IL-10 is a susceptibility factor in VL $[45,46]$. In fact, IL-10 ${ }^{-/-}$mice are highly resistant to L. donovani $[45,47]$. Furthermore, a correlation was found between decreasing levels of IL-10 and resolution of VL in these models. Since IL-10 can act as an inhibitor of IFN- $\gamma$ induced NO synthesis, it is likely that antagonistic effect of IL-10 is mediated by its ability to suppress production of NO, which is critical for elimination of parasites [45].

Various STAT transcription factors appear to be involved in IL-10 production by T cells, B cells, NK cells, monocytes, and macrophages; however, STAT3 plays a conserved role in IL-10 signaling in all cell types [48]. STAT3 is a common transcription factor in signaling by a variety of cytokines, including members of the IL-6 family of cytokines, granulocyte CSF, EGF, IFN- $\gamma$, and IL-2 [49].

STAT3-deficient mice are not viable and experience early fetal death. However, conditional cell-specific knockouts of the STAT3 gene using the Cre-loxP system have demonstrated an important role for STAT3 signaling in IL-6- and IL-2-induced proliferation by $\mathrm{T}$ cells $[23,49]$. In similar studies, IL-10 was demonstrated to play an essential role in the deactivation of macrophages and neutrophils. IL10-deficient mice exhibited increased production of proinflammatory cytokines such as TNF $\alpha$, IL-1, IFN- $\gamma$, and IL6 and a polarized immune response towards a Th1-type response $[23,50]$. The specific role of STAT3 signaling in leishmaniasis has not been demonstrated and requires further investigation.

\section{Conclusion}

The pathology and resolution of leishmaniasis is dependent to a large extent on the infecting species, and the model used. Cytokines do have a role in all cases either by initiating the development of a Th1 response as in VL or development of Th2 2 response in case of CL. The major cytokines in both cases are IL-12, IFN- $\gamma$, IL-10, IL-4, the effects of which are mediated by specific STATs.

\section{Acknowledgment}

Work is Dr. Satoskar's lab is funded by NIH grants AI076309, AI068829.

\section{References}

[1] The Center for Disease Control, "Parasites and health: leishmaniasis," 2008, http://www.dpd.cdc.gov/dpdx/HTML/ Leishmaniasis.htm.

[2] B. L. Herwaldt, "Leishmaniasis," The Lancet, vol. 354, no. 9185, pp. 1191-1199, 1999.

[3] F. J. Tapia, G. Cáceres-Dittmar, and M. A. Sánchez, Molecular and Immune Mechanisms in the Pathogenesis of Cutaneous Leishmaniasis, R. G. Landes, Austin, Tex, USA, 1996.

[4] D. Sacks and A. Sher, "Evasion of innate immunity by parasitic protozoa," Nature Immunology, vol. 3, no. 11, pp. 1041-1047, 2002.

[5] L. E. Rosas, T. Keiser, J. Barbi, et al., "Genetic background influences immune responses and disease outcome of cutaneous L. mexicana infection in mice," International Immunology, vol. 17, no. 10, pp. 1347-1357, 2005.

[6] J. Alexander, A. R. Satoskar, and D. G. Russell, "Leishmania species: models of intracellular parasitism," Journal of Cell Science, vol. 112, no. 18, pp. 2993-3002, 1999.

[7] S. Stäger, J. Alexander, K. C. Carter, F. Brombacher, and P. M. Kaye, "Both interleukin-4 (IL-4) and IL-4 receptor $\alpha$ signaling contribute to the development of hepatic granulomas with optimal antileishmanial activity," Infection and Immunity, vol. 71, no. 8, pp. 4804-4807, 2003.

[8] J. Alexander, K. Christine Carter, N. Al-Fasi, A. Satoskar, and F. Brombacher, "Endogenous IL-4 is necessary for effective drug therapy against visceral leishmaniasis," European Journal of Immunology, vol. 30, no. 10, pp. 2935-2943, 2000.

[9] P. Tripathi, V. Singh, and S. Naik, "Immune response to leishmania: paradox rather than paradigm," FEMS Immunology and Medical Microbiology, vol. 51, no. 2, pp. 229-242, 2007.

[10] D. Sacks and N. Noben-Trauth, "The immunology of susceptibility and resistance to Leishmania major in mice," Nature Reviews Immunology, vol. 2, no. 11, pp. 845-858, 2002.

[11] J. Alexander and K. Bryson, "T helper (h)1/Th2 and Leishmania: paradox rather than paradigm," Immunology Letters, vol. 99, no. 1, pp. 17-23, 2005.

[12] S. G. Reed, "TGF- $\beta$ in infections and infectious diseases," Microbes and Infection, vol. 1, no. 15, pp. 1313-1325, 1999.

[13] P. Kroof, S. Herath, V. Weber, M. Modolell, and I. Müller, "Factors influencing Leishmania major infection in IL-4deficint BALB/c mice," Parasite Immunology, vol. 25, no. 8-9, pp. 439-447, 2003.

[14] L. R. Castellano, D. C. Filho, L. Argiro, et al., "Th1/Th2 immune responses are associated with active cutaneous leishmaniasis and clinical cure is associated with strong interferon$\gamma$ production," Human Immunology, vol. 70, no. 6, pp. 383390, 2009.

[15] L. U. Buxbaum and P. Scott, "Interleukin 10- and Fc $\gamma$ receptordeficient mice resolve Leishmania mexicana lesions," Infection and Immunity, vol. 73, no. 4, pp. 2101-2108, 2005.

[16] B. N. Thomas and L. U. Buxbaum, "Fc $\gamma$ RIII mediates immunoglobulin G-induced interleukin-10 and is required for chronic Leishmania mexicana lesions," Infection and Immunity, vol. 76, no. 2, pp. 623-631, 2008. 
[17] A. L. Wurster, T. Tanaka, and M. J. Grusby, "The biology of Stat4 and Stat6," Oncogene, vol. 19, no. 21, pp. 2577-2584, 2000.

[18] N. G. Jacobson, S. J. Szabo, R. M. Weber-Nordt, et al., "Interleukin 12 signaling in Thelper type 1 (Th1) cells involves tyrosine phosphorylation of signal transducer and activator of transcription (Stat)3 and Stat4," Journal of Experimental Medicine, vol. 181, no. 5, pp. 1755-1762, 1995.

[19] L. E. Rosas, H. M. Snider, J. Barbi, et al., "Cutting edge: STAT1 and T-bet play distinct roles in determining outcome of visceral leishmaniasis caused by Leishmania donovani," Journal of Immunology, vol. 177, no. 1, pp. 22-25, 2006.

[20] J. Bromberg and J. E. Darnell Jr., "The role of STATs in transcriptional control and their impact on cellular function," Oncogene, vol. 19, no. 21, pp. 2468-2473, 2000.

[21] D. J. Ghosh, D. E. Levy, R. W. Johnstone, and C. J. Clarke, "IFN $\gamma$ signaling- does it mean JAK-STAT?" Cytokine \& Growth Factor Reviews, vol. 19, no. 5-6, pp. 282-394, 2008.

[22] D. M. Mosser and J. P. Edwards, "Exploring the full spectrum of macrophage activation," Nature Reviews Immunology, vol. 8, no. 12, pp. 958-969, 2008.

[23] K. Imada and W. J. Leonard, "The Jak-STAT pathway," Molecular Immunology, vol. 37, no. 1-2, pp. 1-11, 2000.

[24] L. E. Rosas, T. Keiser, R. Pyles, J. Durbin, and A. R. Satoskar, "Development of protective immunity against cutaneous leishmaniasis is dependent of STAT1-mediated IFN signaling pathway," European Journal of Immunology, vol. 33, no. 7, pp. 1799-1805, 2003.

[25] H. Bowen, A. Kelly, T. Lee, and P. Lavender, "Control of cytokine gene transcription in Th1 and Th2 cells," Clinical and Experimental Allergy, vol. 38, no. 9, pp. 1422-1431, 2008.

[26] J. Barbi, H. M. Snider, N. Bhardwaj, C.M. Lezama-Dávila, J. E. Durbin, and A. R. Satoskar, "Signal transducer and activator of transcription 1 in $\mathrm{T}$ cells plays an indispensable role in immunity to Leishmania major by mediating Th1 cell homing to the site of infection," The FASEB Journal, vol. 23, no. 11, pp. 3990-3999, 2009.

[27] J. Barbi, S. Oghumu, C. M. Lezama-Davila, and A. R. Satoskar, "IFN- $\gamma$ and STAT1 are required for efficient induction of CXC chemokine receptor 3 (CXCR3) on $\mathrm{CD}^{+}$but not $\mathrm{CD}^{+} \mathrm{T}$ cells," Blood, vol. 110, no. 6, pp. 2215-2216, 2007.

[28] L. E. Rosas, J. Barbi, B. Lu, et al., "CXCR3-/- mice mount an efficient Th1 response but fail to control Leishmania major infection," European Journal of Immunology, vol. 35, no. 2, pp. 515-523, 2005.

[29] L. M. Johnson and P. Scott, "STAT1 expression in dendritic cells, but not T cells, is required for immunity to Leishmania major," Journal of Immunology, vol. 178, no. 11, pp. 72597266, 2007.

[30] A. Diefenbach, H. Schindler, N. Donhauser, et al., "Type I interferons $(\operatorname{IFN} \alpha / \beta)$ and type 2 nitric oxide synthase regulate the innate immune response to a protozoan parasite," Immunity, vol. 8, no. 1, pp. 77-87, 1998.

[31] L. Xin, K. Li, and L. Soong, "Down-regulation of dendritic cell signaling pathways by Leishmania amazonensis amastigotes," Molecular Immunology, vol. 45, no. 12, pp. 3371-3382, 2008.

[32] G. Trinchieri, "Interleukin-12 and the regulation of innate resistance and adaptive immunity," Nature Reviews Immunology, vol. 3, no. 2, pp. 133-146, 2003.

[33] W. T. Watford, B. D. Hissong, J. H. Bream, Y. Kanno, L. Muul, and J. J. O'Shea, "Signaling by IL-12 and IL-23 and the immunoregulatory roles of STAT4," Immunological Reviews, vol. 202, pp. 139-156, 2004.
[34] M. A. McDowell and D. L. Sacks, "Inhibition of host cell signal transduction by Leishmania: observations relevant to the selective impairment of IL-12 responses," Current Opinion in Microbiology, vol. 2, no. 4, pp. 438-443, 1999.

[35] A. R. Satoskar, S. Rodig, S. R. Telford III, et al., "IL-12 gene-deficient C57BL/6 mice are susceptible to Leishmania donovani but have diminished hepatic immunopathology," European Journal of Immunology, vol. 30, no. 3, pp. 834-839, 2000.

[36] M. Rodriguez-Sosa, G. M. Monteforte, and A. R. Satoskar, "Susceptibility to Leishmania mexicana infection is due to the inability to produce IL-12 rather than lack of IL-12 responsiveness," Immunology and Cell Biology, vol. 79, no. 4, pp. 320-322, 2001.

[37] K. Nelms, H. Huang, J. Ryan, A. Keegan, and W. E. Paul, "The IL-4 receptor: signaling mechanisms and biological functions," Advances in Experimental Medicine and Biology, vol. 452, pp. 37-43, 1998.

[38] D. Hebenstreit, G. Wirnsberger, J. Horejs-Hoeck, and A. Duschl, "Signaling mechanisms, interaction partners, and target genes of STAT6," Cytokine and Growth Factor Reviews, vol. 17, no. 3, pp. 173-188, 2006.

[39] A. Satoskar, H. Bluethmann, and J. Alexander, "Disruption of the murine interleukin- 4 gene inhibits disease progression during Leishmania mexicana infection but does not increase control of Leishmania donovani infection," Infection and Immunity, vol. 63, no. 12, pp. 4894-4899, 1995.

[40] C. R. Engwerda, S. C. Smelt, and P. M. Kaye, "An in vivo analysis of cytokine production during Leishmania donovani infection in scid mice," Experimental Parasitology, vol. 84, no. 2, pp. 195-202, 1996.

[41] M. E. Wilson, B. M. Young, B. L. Davidson, K. A. Mente, and S. E. McGowan, "The importance of TGF- $\beta$ in murine Visceral leishmaniasis," Journal of Immunology, vol. 161, no. 11, pp. 6148-6155, 1998.

[42] S. Stäger, J. Alexander, A. C. Kirby, et al., "Natural antibodies and complement are endogenous adjuvants for vaccineinduced $\mathrm{CD}^{+}$T-cell responses," Nature Medicine, vol. 9, no. 10 , pp. 1287-1292, 2003.

[43] L. U. Buxbaum, "A detrimental role for IgG and FcgammaR in Leishmania mexicana infection," Immunologic Research, vol. 42, no. 1-3, pp. 197-209, 2008.

[44] S. Bhowmick, T. Mazumdar, and N. Ali, "Vaccination route that induces transforming growth factor $\beta$ production fails to elicit protective immunity against Leishmania donovani infection," Infection and Immunity, vol. 77, no. 4, pp. 15141523, 2009.

[45] M. L. Murphy, U. Wille, E. N. Villegas, C. A. Hunter, and J. P. Farrell, "IL-10 mediates susceptibility to Leishmania donovani infection," European Journal of Immunology, vol. 31, no. 10, pp. 2848-2856, 2001.

[46] H. W. Ghalib, M. R. Piuvezam, Y. A. W. Skeiky, et al., "Interleukin 10 production correlates with pathology in human Leishmania donovani infections," Journal of Clinical Investigation, vol. 92, no. 1, pp. 324-329, 1993.

[47] D. Nandan and N. E. Reiner, "Leishmania donovani engages in regulatory interference by targeting macrophage protein tyrosine phosphatase SHP-1," Clinical Immunology, vol. 114, no. 3, pp. 266-277, 2005.

[48] D. M. Mosser and X. Zhang, "Interleukin-10: new perspectives on an old cytokine," Immunological Reviews, vol. 226, no. 1, pp. 205-218, 2008. 
[49] K. Takeda, T. Tsuneyasuk, N. Yoshida, J. Takeda, T. Kishimoto, and S. Akira, "Stat3 activation is responsible for IL-6dependent $\mathrm{T}$ cell proliferation through preventing apoptosis: generation and characterization of T-cell- specific stat3deficient mice," Journal of Immunology, vol. 161, no. 9, pp. 4652-4660, 1998.

[50] K. Takeda, B. E. Clausen, T. Kaisho, et al., "Enhanced Th1 activity and development of chronic enterocolitis in mice devoid of STAT3 in macrophages and neutrophils," Immunity, vol. 10, no. 1, pp. 39-49, 1999. 

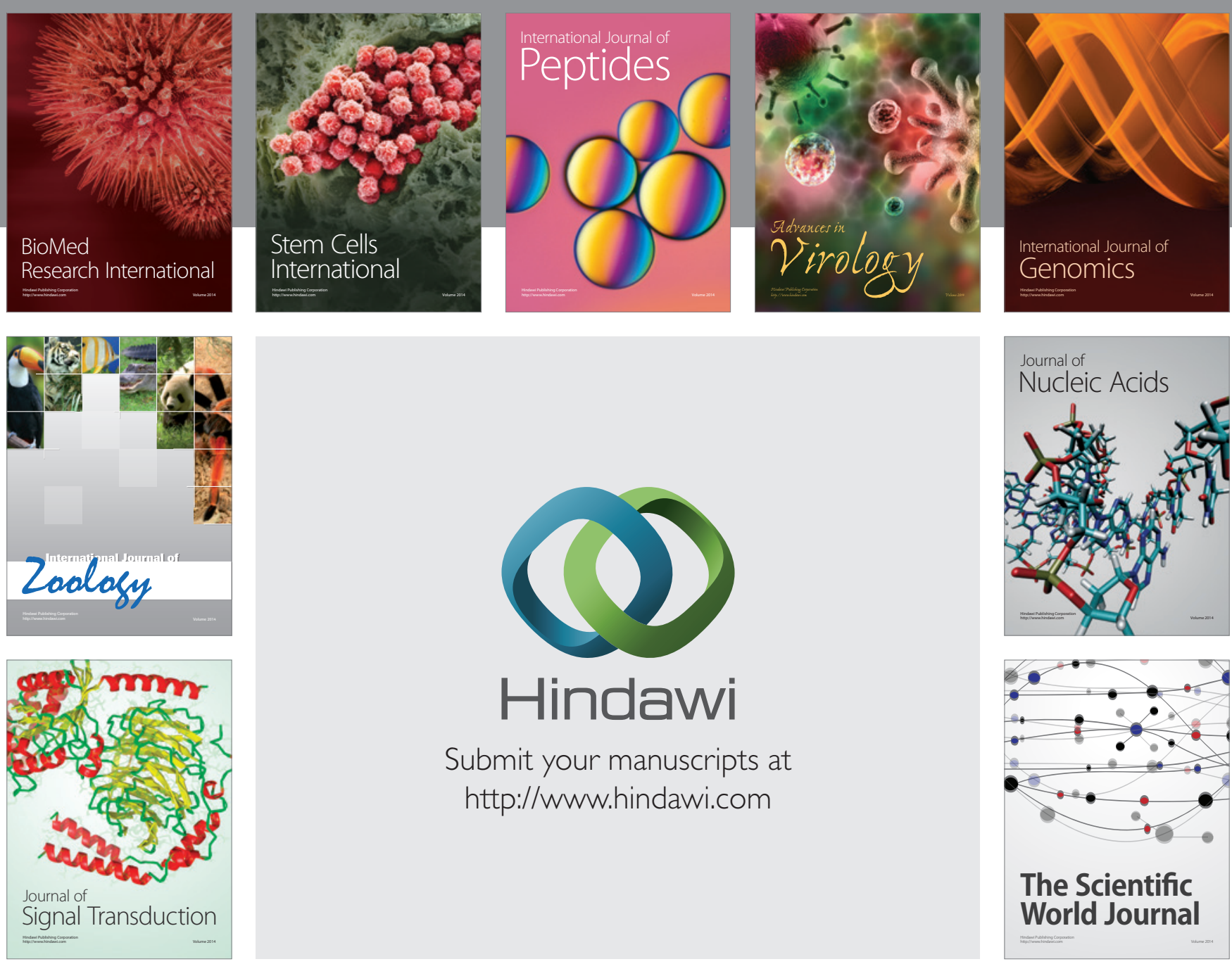

Submit your manuscripts at

http://www.hindawi.com
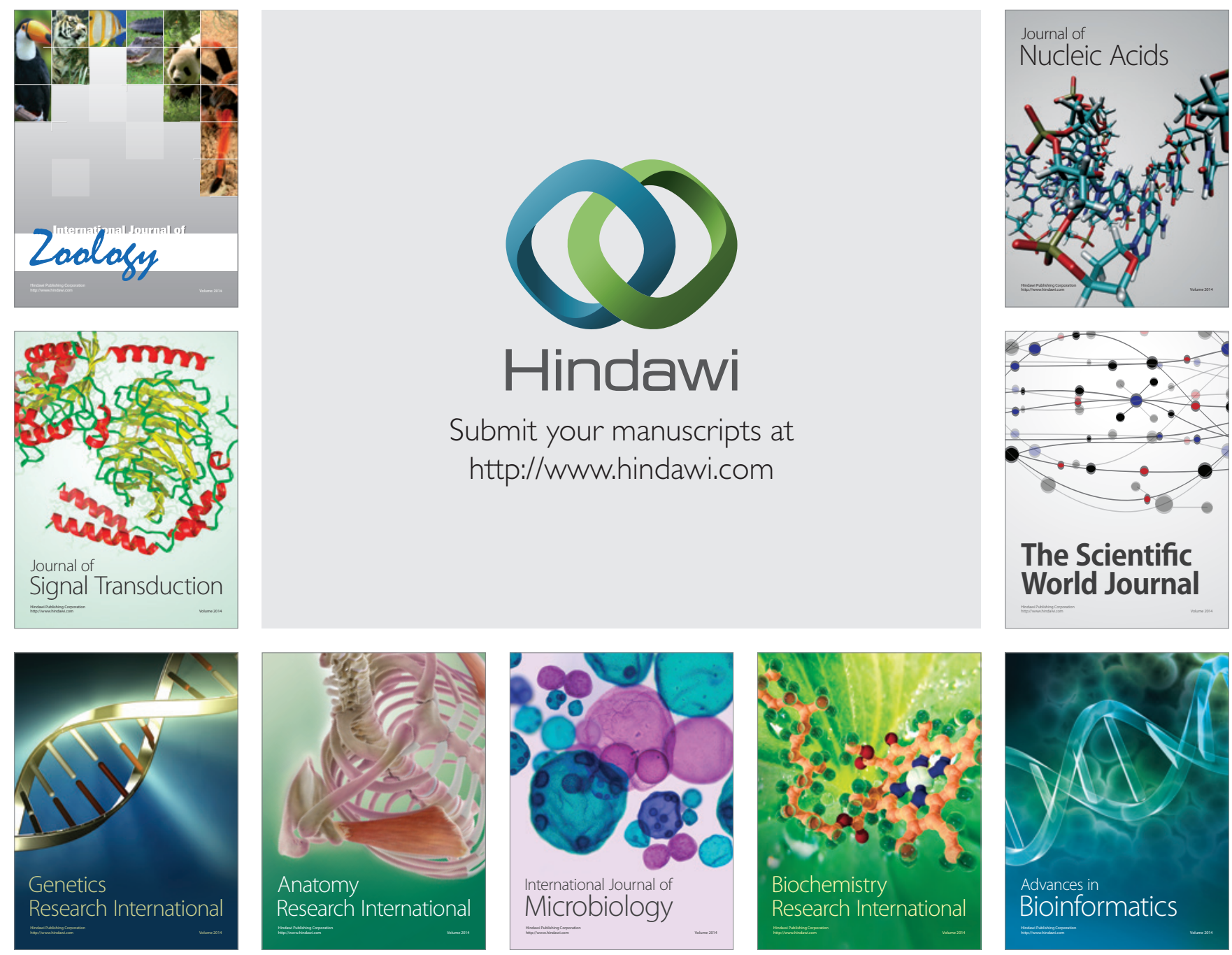

The Scientific World Journal
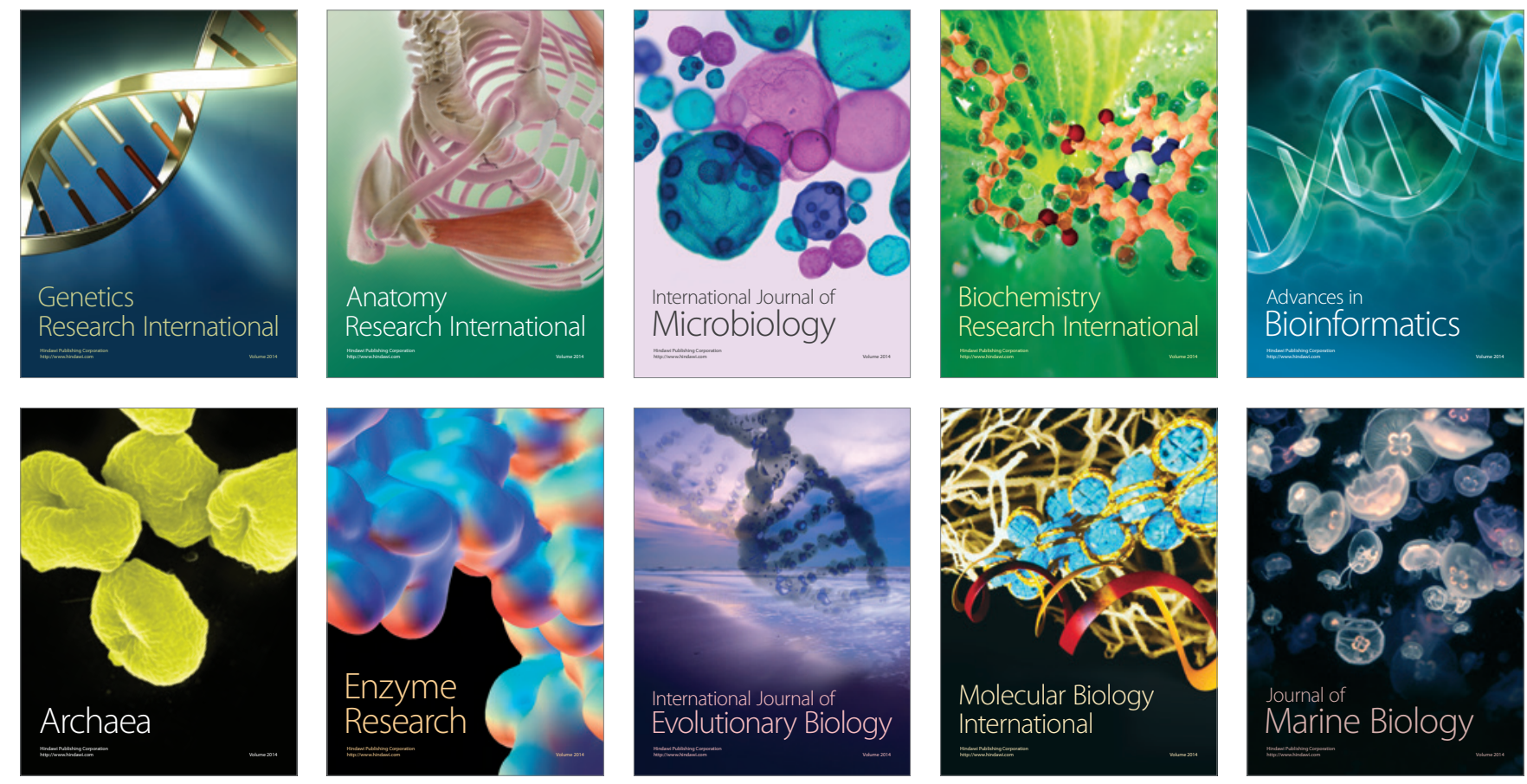